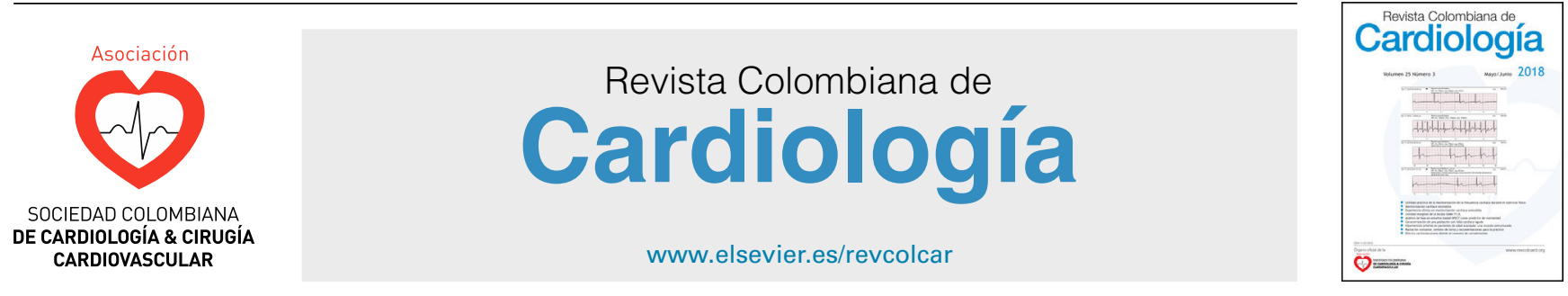

CIRUGÍA CARDIOVASCULAR DEL ADULTO-PRESENTACIÓN DE CASOS

\title{
Evaluación de resultados en una serie de pacientes con reparación de aneurimas aórticos mediante endoprótesis fenestradas
}

\author{
Juan F. Gómez-Hoyos ${ }^{\mathrm{a}}$, Juan G. Muñoz Sierra ${ }^{\mathrm{a}}$, Camila Lema-Calidonio ${ }^{\mathrm{a}, *}$, \\ Sebastián Calle-Díaz ${ }^{b}$ y Nathalia González-Jaramillo ${ }^{a}$
}

\author{
${ }^{a}$ Clínica Cardio VID, Medellín, Colombia \\ b Facultad de Medicina, Universidad Pontificia Bolivariana, Medellín, Colombia
}

Recibido el 4 de septiembre de 2017; aceptado el 24 de octubre de 2018

Disponible en Internet el 8 de junio de 2019

\section{PALABRAS CLAVE \\ Enfermedades cardiovasculares; Aneurisma; Endoprótesis.}

\begin{abstract}
Resumen
Introducción: el aneurisma de aorta tiene una prevalencia de 5,7\%; sin tratamiento su desenlace final es la ruptura y en ocasiones la muerte. En la actualidad, existe el reparo endovascular mediante prótesis fenestradas para pacientes en quienes es inviable el procedimiento quirúrgico. Están diseñadas para adaptarse a la anatomía única de cada paciente y dan cabida al flujo de uno o más vasos viscerales logrando resultados satisfactorios.

Objetivo: reportar una serie de casos de pacientes con aneurismas de aorta tratados mediante endoprótesis fenestradas en un centro de referencia cardiovascular de Medellín, Colombia, entre 2013 y 2017.

Método: estudio descriptivo, restrospectivo, de una serie de casos, cuya información se obtuvo por medio de una fuente secundaria.

Resultados: los resultados se presentaron como porcentajes y frecuencias. Se utilizó el programa SPSS versión 20.0. La mayoría de los pacientes fueron hombres, con promedio de edad de $69,5 \pm 7,9$ años.

La mediana del diámetro aneurismático fue de 6,0 cm (RIQ 5,0-8,25). La mayoría de las complicaciones fueron vasculares. El $80,0 \%$ de los pacientes tuvieron algún seguimiento radiológico al mes, 6 meses o a los 12 meses después de la intervención; el 100,0\% requirió revascularización del o los vasos intervenidos.

Conclusiones: se considera que el balance adecuado entre riesgos y beneficios que otorga la técnica hace que, para el grupo específico de pacientes cuyas características clínicas y anatómicas hacen poco factible la cirugía, el uso de las prótesis fenestradas sea seguro y tenga resultados clínicos y de supervivencia similares al compararse con la literatura.

(C) 2019 Sociedad Colombiana de Cardiología y Cirugía Cardiovascular. Publicado por Elsevier España, S.L.U. Este es un artículo Open Access bajo la licencia CC BY-NC-ND (http:// creativecommons.org/licenses/by-nc-nd/4.0/).
\end{abstract}

\footnotetext{
* Autor para correspondencia.

Correo electrónico: clema@vid.org.co (C. Lema-Calidonio).
} 


\section{KEYWORDS}

Cardiovascular

diseases;

Aneurysm;

Stent graft.

\section{Evaluation of results in a series of patients with repair of aortic aneurims by fenestrated graft stent}

\begin{abstract}
Introduction: Aortic aneurysm has a prevalence of $5.7 \%$. Its final outcome without is a rupture, and occasionally death. Endovascular repair can currently be carried out using a fenestrated stent graft in patients in whom a surgical procedure is not feasible. These grafts are designed to adapt to the unique anatomy of each patient, and makes room for one or more visceral vessels to flow, achieving satisfactory results.

Objective: To report a case series of patients with aortic aneurysms treated with a fenestrated stent graft between 2013 and 2017 in a cardiovascular reference centre in Medellín, Colombia. Method: A descriptive, retrospective study was conducted on a case series, in which the information was obtained for a secondary source. The results are presented as percentages and frequencies. The statistics program SPSS version 20.00 was used.

Results: The majority of patients were male, and the mean age was $69.5 \pm 7.9$ years. The median aneurysm diameter was $6.0 \mathrm{~cm}$ (IQR 5.0-8.25). The majority of complications were vascular. Some type of radiological follow-up was carried out on $80 \%$ of the patients at one month, 6 months, and 12 months after the intervention. All of them (100\%) required revascularisation of the vessels intervened.

Conclusions: The risk-benefit ratio is considered adequate for this technique. It means that, for the specific group of patients that have clinical and anatomical characteristics that make surgery highly unlikely, the use of fenestrated stent grafts are safe, and have similar clinical and survival results when compared with the literature.

(c) 2019 Sociedad Colombiana de Cardiología y Cirugía Cardiovascular. Published by Elsevier España, S.L.U. This is an open access article under the CC BY-NC-ND license (http:// creativecommons.org/licenses/by-nc-nd/4.0/).
\end{abstract}

\section{Introducción}

La enfermedad cardiovascular es la principal causa de morbilidad y mortalidad en el mundo; cada año produce alrededor de 17,3 millones de muertes ${ }^{1}$ y está estrechamente relacionada con diferentes factores de riesgo, entre los cuales figuran la hipertensión arterial, el tabaquismo, el consumo de alcohol, la dieta y la obesidad, entre otros. Dicha enfermedad se puede manifestar en cuatro categorías: enfermedad coronaria, enfermedad cerebrovascular, enfermedad arterial periférica y aterosclerosis/aneurisma de la aorta. Los aneurismas de la aorta son una enfermedad causada por la dilatación permanente de las paredes de dicha arteria. Su incidencia anual está calculada en 40 casos por cada 100 personas cuando se trata de aneurismas abdominales y en 10 a 15 casos por cada 100 personas para los aneurismas torácicos. Su prevalencia conjunta oscila alrededor del $5,7 \%^{2}$. Esta enfermedad es más frecuente en hombres que en mujeres y tiene un pico de presentación a la edad de 70 años $^{3}$. El desenlace final es la ruptura, la cual depende principalmente del diámetro del aneurisma y puede llevar a la muerte. Los aneurismas de la aorta se clasifican de dos maneras diferentes según su localización: aneurismas de la aorta torácica y aneurismas de la aorta abdominal; esta última es responsable de al menos tres cuartas partes de todos los aneurismas aórticos.

La enfermedad tiene altos niveles de mortalidad y se ha asociado con los principales factores de riesgo conocidos para la enfermedad cardiovascular en general; sin embargo, particularmente, los que mayor peso tienen para desarrollarla son la hipertensión y los procesos ateroscleróticos, para ATT y para aneurismas de la aorta abdominal respectivamente ${ }^{4}$.

Dada la alta mortalidad de los aneurismas de la aorta abdominal, en la que $50,0 \%$ de los pacientes con ruptura del aneurisma que llegan al hospital para recibir tratamiento, entre el 30,0 y el 50,0\% morirán en el hospital ${ }^{5}$. El manejo médico se reserva para los pacientes asintomáticos con aneurismas pequeños menores de $5,5 \mathrm{~cm}$ o pacientes inoperables ${ }^{6}$. Aunque la cirugía abierta fue durante décadas la terapia estándar, a partir del año 2005 la Administración de Medicamentos y Alimentos de Estados Unidos (FDA su sigla en inglés), aprobó la utilización de endoprótesis como tratamiento de reparo endovascular para los aneurismas aórticos. Con el paso del tiempo, dicho procedimiento ha tomado cada vez más fuerza en los últimos años gracias al balance aceptable entre su seguridad y eficacia. Algunos estudios indican mejores resultados para el paciente con los nuevos procedimientos de reparación endovascular ${ }^{7}$. Los beneficios que se evidencian con estos últimos son: menor tasa de mortalidad perioperatoria $^{8}$, no requerimiento de esternotomía, toracotomía o incisiones de gran tamaño en la región abdominal, no pinzamiento de la aorta, menor riesgo de sangrado, menor incidencia de isquemia en órganos diana, menor necesidad de ayudas externas respiratorias, y proceso de recuperación más corto ${ }^{9-11}$.

Si bien se plantean muchos beneficios a favor de la reparación endovascular, también hay factores en contra como la trombosis de la endoprótesis o endofugas de la misma. En consecuencia, es necesario decidir el mejor tratamiento 
para el paciente, con base en un enfoque individualizado antes del procedimiento, en el cual se deben tener en cuenta edad, factores de riesgo, anatomía propia del paciente, posible morbilidad y mortalidad perioperatorias, bien sea para elegir la cirugía abierta o el procedimiento de reparación endovascular. Los procedimientos mencionados están indicados para los pacientes sintomáticos y asintomáticos con aneurisma de expansión rápida o un diámetro mayor de 5 a $6 \mathrm{~cm}$ (dependiendo del tamaño de la contextura del paciente $)^{12,13}$. Para aquellos con aneurismas de aorta abdominal que comprometen las bifurcaciones de las arterias renales o mesentéricas, o cuyos cuellos cortos impiden una adecuada implantación de una prótesis convencional, se cuenta desde el año 2012 con aprobación por parte de la Administración de medicamentos y alimentos, para la comercialización y utilización de endoprótesis fenestradas, si bien el primer caso en humanos con este tipo de prótesis data de 1996.

Estas prótesis constan de un sistema modular que tiene tres componentes: un cuerpo proximal, un cuerpo bifurcado distal, una rama ilíaca y en estos componentes, aberturas que permiten la salida de las arterias renales y viscerales. Las endoprótesis fenestradas están diseñadas para adaptarse a la anatomía única de cada paciente, ya que son hechas a medida de acuerdo con las variaciones individuales, y permiten el flujo de uno o más de los vasos viscerales ${ }^{14}$. En cuanto a anatomía, el límite absoluto para el implante de una endoprótesis estándar es la presencia de una o ambas arterias renales emergiendo del saco aneurismático. Cuando esto ocurre, la única posibilidad de excluir el aneurisma es por vía endovascular con una endoprótesis fenestrada que preserve el flujo sanguíneo hacia dichas arterias ${ }^{15}$.

Pese a que existen reportes en la literatura mundial desde hace al menos una década, en Colombia no hay hasta el momento publicaciones que reporten la experiencia con el uso de estas prótesis. El objetivo de este trabajo es reportar una serie de casos de pacientes consecutivos tratados mediante endoprótesis fenestradas en un centro de referencia cardiovascular de la ciudad de Medellín desde el inicio de la implementación de la técnica en enero de 2013 hasta el mes de junio de 2017.

\section{Métodos}

Se llevó a cabo la descripción y el análisis de una serie de casos de pacientes con aneurismas de aorta abdominal y torácica que fueron tratados mediante endoprótesis fenestradas en un centro de referencia cardiovascular de la ciudad de Medellín, desde el inicio de la incorporación de la técnica a partir de enero de 2013 hasta 2017.

Una vez se realizó el diagnóstico de aneurisma aórtico abdominal yuxtarrenal (AAAY) o aneurisma aórtico toracoabdominal (AATA) para cada paciente, se evaluó la opción terapéutica entre el equipo de Cirugía Cardiovascular junto con Radiología Intervencionista de la institución según la viabilidad y el riesgo- beneficio de la intervención; luego, al decidir que la endoprótesis era la mejor opción terapéutica, se solicitó al proveedor construirla según la medida anatómica de cada paciente.

Estos procedimientos se llevaron a cabo bajo anestesia general. Durante la intervención se dispuso de cirujano cardiovascular; se utilizó la endoprótesis fenestrada de COOK Medical Inc. Un Radiólogo Intervencionista la insertó y posterior a esto se tomó un aortograma de control para garantizar el posicionamiento adecuado de la prótesis. Al salir del procedimiento los pacientes fueron tratados en la Unidad de Cuidados Intensivos ( $\mathrm{UCI}$ ) inicialmente.

A partir de la fecha del reparo endovascular los Radiólogos Intervencionistas hicieron seguimiento de los pacientes con ayuda de imágenes de control al mes, a los 6 meses, a los 12 meses en la medida de lo posible. Se obtuvo la información de una fuente secundaria proveniente de la base de datos de registros de estos pacientes de la institución. Los datos se recolectaron de forma retrospectiva.

Se llevó a cabo una revisión extensa de cada paciente acerca de las características sociodemográficas, factores predisponentes, características del aneurisma, estancia hospitalaria, indicaciones, complicaciones del procedimiento, así como seguimiento por parte de médico tratante (radiólogo intervencionista), seguimiento imagenológico, que incluye los desenlaces críticos como fugas, necesidad de reintervención, permeabilidad de los vasos, crecimiento aórtico y secuelas por causa de complicaciones durante el procedimiento. El éxito de la intervención se evaluó respecto a la necesidad de reintervención a los 12 meses y con la permeabilidad de los vasos.

Para hacer el análisis estadístico se utilizó el programa SPSS versión 20.0. Todas las variables cualitativas se reportan mediante frecuencias absolutas y relativas, mientras que el resumen de las variables cuantitativas se llevó a cabo con medidas de tendencia central y dispersión, que fueron elegidas según la distribución de cada una de las variables, así: para las variables con distribución normal se calcularon promedio y desviación estándar y para aquellas que no se ajustaron a dicha distribución, se calcularon mediana y rango intercuartílico (RIQ).

Se llevaron a cabo pruebas de Chi cuadrado para evaluar la posible relación entre variables de exposición de interés y desenlaces de mortalidad e isquemia mesentérica. Para todos los casos de análisis de relación entre múltiples variables, se consideró un error alfa máximo del $5 \%$ y una confiabilidad del $95 \%$.

\section{Resultados}

Entre los años 2013 a 2017 se realizaron 30 procedimientos de reparación endovascular de aneurisma aórtico mediante la utilización de endoprótesis fenestradas, en un centro de referencia cardiovascular de la ciudad de Medellín.

La mayoría de pacientes sometidos a esta intervención fueron hombres con promedio de edad de 69,5 $\pm 7,9$ años. En cuanto a los factores predisponentes para desarrollar aneurisma de aorta, se observó que los más frecuentes fueron hipertensión arterial (HTA) seguido de tabaquismo. En el $40,0 \%$ de los casos ya se había realizado una reparación quirúrgica de dicho aneurisma en años anteriores (tabla 1).

Para el grupo analizado, la mediana del diámetro aneurismático fue de $6,0 \mathrm{~cm}$ (RIQ 5,0-8,25).

La localización más habitual del aneurisma fue la aorta abdominal (90,0\%). Respecto a las ramas afectadas, el $76,7 \%$ de los pacientes tuvo compromiso a nivel del origen de las arterias mesentéricas, el $86,7 \%$ en origen de las arterias 
Tabla 1 Características demográficas y antecedentes personales

\begin{tabular}{lll}
\hline Variable & Frecuencia & Porcentaje \\
\hline Sexo masculino & 18 & $60,0 \%$ \\
Hipertensión arterial & 26 & $86,7 \%$ \\
Diabetes mellitus & 7 & $23,3 \%$ \\
Dislipidemia & 23 & $76,7 \%$ \\
Insuficiencia renal crónica & 5 & $16,7 \%$ \\
Tabaquismo & 24 & $80,0 \%$ \\
Enfermedad coronaria & 11 & $36,7 \%$ \\
Enfermedad carotídea & 5 & $16,7 \%$ \\
Reparo quirúrgico & 12 & $40,0 \%$ \\
\hline
\end{tabular}

Tabla 2 Complicaciones derivadas de la intervención

\begin{tabular}{|c|c|c|c|}
\hline \multirow[b]{2}{*}{$\begin{array}{l}\text { Complicaciones } \\
\text { vasculares }\end{array}$} & \multicolumn{2}{|c|}{ Resultados propios } & \multirow{2}{*}{$\frac{\text { Literatura }^{16,1}}{23,30 \%}$} \\
\hline & 4 & $13,30 \%$ & \\
\hline Isquemia renal & 3 & $10,00 \%$ & $6,70 \%$ \\
\hline Isquemia mesentérica & 1 & $3,30 \%$ & $3,30 \%$ \\
\hline Isquemia medular & 3 & $10,00 \%$ & $0,00 \%$ \\
\hline $\begin{array}{l}\text { Infección } \\
\text { intrahospitalaria }\end{array}$ & 4 & $13,30 \%$ & $3,30 \%$ \\
\hline Muerte intrahospitalaria & 2 & $6,70 \%$ & $4,30 \%$ \\
\hline Reingreso & 7 & $23,30 \%$ & $\mathrm{NR}^{*}$ \\
\hline $\begin{array}{l}\text { Muerte al año de } \\
\text { seguimiento }\end{array}$ & 1 & $3,30 \%$ & $6,60 \%$ \\
\hline
\end{tabular}

*NR: No registra.

renales y el $10,0 \%$ en origen de las arterias carótidas. El $76,7 \%$ de los procedimientos realizados fueron de carácter electivo.

En la tabla 2 se exponen las complicaciones de la intervención y se hace una comparación con las complicaciones reportadas por la literatura. Se encontró que el 13,3\% presentó complicaciones vasculares (ruptura de la arteria humeral izquierda, oclusión de arteria femoral izquierda y trombosis de la arteria femoral) y el $10,0 \%$ isquemia medular. Fallecieron $10,0 \%$ de los pacientes, uno a causa de síndrome de disfunción multiorgánica que ocasionó choque vasopléjico refractario al manejo en Unidad de Cuidados UCI y dos por choque hipovolémico secundario a isquemia mesentérica. La mediana de estancia hospitalaria fue de 5,0 (RIQ 4,0-12,5) días y en $\mathrm{UCl}$ fue de 3,0 (RIQ 2,0-4,0) días. Las causas de reingresos relacionadas con la intervención fueron: dolor neuropático en miembro inferior izquierdo, dolor inguinal derecho y respuesta inflamatoria sistémica postprocedimiento o síndrome postimplantación.

Al evaluar el seguimiento por Radiología Intervencionista se encontró que no se dispone de información adicional de 3 $(10,0 \%)$ pacientes posterior a la intervención y 3 pacientes fallecieron como se mencionó anteriormente, ninguno de ellos alcanzó seguimiento. 24 (80,0\%) tuvieron algún seguimiento: $91,7 \%$ al mes, $70,8 \%$ a los seis meses y $54,2 \%$ a los doce meses a partir de la fecha del procedimiento. El 50,0\% tuvo los tres seguimientos completos.

Adicionalmente, se evaluó el control por imagen por medio de tomografía axial computarizada (TAC) simple de abdomen $(41,6 \%)$, Doppler aórtico $(29,2 \%)$ o AngioTAC
$(29,2 \%)$. Según el último registro de seguimiento que se obtuvo de cada paciente, se observó que el $12,5 \%$ tuvo crecimiento de la aorta; para ello se definió seguimiento con imagen cada 6 meses inicialmente, ya que no se consideró crecimiento clínicamente significativo para reintervenir; $100,0 \%$ requirió revascularización del o los vasos intervenidos a los diferentes momentos de control y el $13,3 \%$ tuvo fugas (todas clasificadas como tipo II) un caso de los cuales $(10,0 \%)$ requirió reintervención a los 36 meses del procedimiento; este paciente corresponde al primer caso que se trató en la institución en el año 2013.

Luego de la comparación Chi cuadrado para evaluar la posible relación de las variables con el desenlace de mortalidad intrahospitalaria, se encontró que el paciente que presentó como complicación isquemia mesentérica, falleció. Así, mientras la mortalidad fue del $100 \%$ respecto a esta complicación, la mortalidad en quienes no la desarrollaron fue del 3,4\%. Dicha diferencia fue estadísticamente significativa a un valor de $\mathrm{p} 0,006$. No hubo asociación con ninguna otra de las variables analizadas.

Respecto a las infecciones, que se hallaron en una proporción más alta que la reportada por la literatura, desde el comité de infecciones se analizó que de los 4 casos, 3 tenían infección asociada a la atención de la salud ( 2 casos de infección urinaria asociada a sonda vesical y un caso de bacteriemia secundaria a infección intraabdominal por isquemia mesentérica). El caso restante correspondió a una colecistitis asociada a colelitiasis, que no estuvo relacionado ni con el procedimiento ni con ninguna de las actividades realizadas en el marco de la atención de la salud. Ninguno de los casos se consideró infección del sitio operatorio relacionado con el procedimiento.

Debido a la importancia de la isquemia mesentérica en el desenlace de mortalidad y a su relación con las infecciones intrahospitalarias, se evaluó la relación de otras variables con este desenlace y se encontró que los pacientes con antecedentes de insuficiencia renal tuvieron una probabilidad neta del $33,0 \%$ de presentar isquemia mesentérica en comparación con la probabilidad del $0,0 \%$ para quienes no tenían dicho antecedente. Esta diferencia fue estadística-

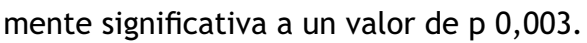

\section{Discusión}

Aunque los aneurismas de la aorta constituyen una enfermedad con una prevalencia relativamente alta, y con altos niveles de mortalidad si no es tratada ${ }^{2}$, en Colombia no hay hasta el momento publicaciones con el reporte de la experiencia de uso de prótesis endovasculares fenestradas para el tratamiento de esta enfermedad. Por esta razón, se presenta el reporte de una serie de casos en una población de pacientes sometidos a este procedimiento, desde el inicio de uso de la técnica en el año 2013 hasta junio de 2017 en un centro de referencia cardiovascular de la ciudad de Medellín.

En este estudio se encontraron similitudes con lo reportado por la literatura en cuanto a antecedentes y datos sociodemográficos tales como mayor representación del sexo masculino, edad promedio mayor a 65 años, y prevalencia alta de hipertensión arterial y tabaquismo ${ }^{3,4}$. 
Como era también de esperarse según la distribución de incidencias, la mayor proporción de los pacientes tratados tuvo compromiso de la aorta abdominal ${ }^{12}$. Por tratarse de una serie de pacientes intervenidos mediante endoprótesis fenestradas, se reporta el compromiso de las ramas arteriales de las cuales las arterias renales y mesentéricas fueron las más frecuentes. A pesar de tratarse de una población de edad no muy avanzada, se encontró que un $40,0 \%$ de los pacientes ya habían tenido en años anteriores una intervención quirúrgica previa dirigida al tratamiento de su aneurisma.

Es importante anotar que las endoprótesis fenestradas constituyen una alternativa importante a la cirugía abierta $^{7}$; sin embargo, muchos centros están incrementando de manera progresiva su oferta como parte del estándar de tratamiento en la enfermedad aórtica. Los últimos veinte años han mostrado rápidos avances hasta el punto que los resultados de estas prótesis pueden competir con los de la cirugía convencional ${ }^{9,11}$.

Para la serie de pacientes que aquí se reporta en comparación con la literatura referente a las endoprótesis fenestradas, se encontró menor tasa de complicaciones vasculares luego del procedimiento, así como menor mortalidad al año de seguimiento y menos pacientes con fuga durante ese mismo tiempo $13,3 \%$ vs. $26,0 \%$ para reparo endovascular reportado en la literatura vs. una incedencia del 10 al $50 \%$ con la cirugía convencional ${ }^{18}$.

Se destaca que en el análisis de mortalidad intrahospitalaria, se halló que la isquemia mesentérica fue el único factor asociado con la mortalidad luego del procedimiento y que ésta ocurrió únicamente en el paciente con antecedentes de insuficiencia renal moderada a severa. Estudios previos han mostrado que la insuficiencia renal preexistente aumenta la mortalidad periprocedimiento hasta en ocho veces $^{19}$. Los hallazgos obtenidos, así como lo reportado por la literatura al respecto, sugieren que si bien el antecedente de insuficiencia renal en sí mismo no debe ser un criterio de exclusión para recibir la intervención, es necesaria una selección cuidadosa de los pacientes, que incluya no sólo las características propias del aneurisma sino también la consideración de todos los desenlaces adversos perioperatorios posibles en función de las enfermedades de base.

Luego de analizarse la causalidad de las infecciones postprocedimiento, el comité de infecciones institucional determinó que en los casos revisados se encontró adherencia total a los protocolos de profilaxis antimicrobiana y medidas para evitar infección en relación con asepsia y antisepsia. Pese a que el síndrome postimplantación está bien descrito y es así como algunos pacientes luego del procedimiento pueden presentar fiebre, leucocitosis e incluso infiltrados pulmonares no infecciosos en el postoperatorio, en los casos analizados como infecciones no hubo esta discrepancia diagnóstica ya que un solo paciente que lo presentó no fue incluido dentro de las infecciones.

A partir de la experiencia institucional, se recomienda que una vez se sospeche un caso de infección asociada a la atención de la salud (IAAS), el caso sea revisado y discutido por un equipo que defina:

- Si de acuerdo con los criterios del Instituto Nacional de Salud (INS) y del Centro de Control y Prevención de
Enfermedades (CDC por su sigla en inglés: Centers for Disease Control and Prevention) es una IAAS.

- Según el protocolo de Londres cuáles fueron los factores del paciente, de la atención y del ambiente que pudieron haber influido en la aparición de infecciones.

- Si hubo o no fallas activas, a partir de la evitabilidad o no de los eventos.

Por último, el seguimiento se considera fundamental para evaluar el resultado de la intervención y lo ideal es que médicos tratantes son quienes deben hacerlo; sin embargo, se debe tener en cuenta que por el sistema de salud del país es difícil que los seguimientos se realicen exactamente en el tiempo estipulado y en ocasiones por cambios administrativos los pacientes deben consultar a otra institución para su seguimiento. Esto explica que solamente la mitad de los pacientes fueron evaluados al mes, 6 y 12 meses después de la intervención y que a pesar de dichas dificultades la mayoría tuvieron seguimiento en algún momento posterior al procedimiento.

Adicionalmente, tomar los datos en forma retrospectiva como se hizo en este estudio, es una limitante.

En conclusión, y a partir de la experiencia adquirida en este centro, se considera que el adecuado balance entre riesgos y beneficios que otorga la técnica, hace que para el grupo específico de pacientes cuyas características clínicas y anatómicas hacen poco factible la cirugía, el uso de las prótesis fenestradas presenta buenos resultados clínicos y de supervivencia al año al compararse con la literatura.

Es probable que la investigación continua en nuevos dispositivos y técnicas permita mejorar los actuales resultados.

\section{Financiación}

Ninguna.

\section{Conflicto de interés}

Ninguno.

\section{Bibliografía}

1. Laslett LJ, Alagona P, Clark BA, Drozda JP, Saldivar F, Wilson SR, et al. The worldwide environment of cardiovascular disease: prevalence, diagnosis, therapy, and policy issues. J Am Coll Cardiol. 2012;60:S1-49.

2. Stather PW, Sidloff DA, Rhema IA, Choke E, Bown MJ, Sayers RD. A review of current reporting of abdominal aortic aneurysm mortality and prevalence in the literature. Eur J Vasc Endovasc Surg. 2014;47:240-2.

3. Arrieta M, Marzola K. Efectividad del tratamiento endovascular de aneurismas aórticos torácicos y abdominales en Cartagena. Colombia. Rev Cienc Bioméd. 2015;6:60-7.

4. Kumar V, Robbins SL, Cotran RS, editores. Robbins and Cotran pathologic basis of disease: [expertconsult, searchable full text online]. professional ed. Philadelphia, Pa: Elsevier 8: Saunders; 2010. p. p.1450.

5. Bown MJ, Sutton AJ, Bell PR, Sayers RD. A meta-analysis of 50 years of ruptured abdominal aortic aneurysm repair. Br J Surg. 2002;89:714-30. 
6. Johnston KW1, Rutherford RB, Tilson MD, Shah DM, Hollier L, Stanley JC. Suggested standards for reporting on arterial aneurysms. Subcommittee on Reporting Standards for Arterial Aneurysms, Ad Hoc Committee on Reporting Standards, Society for Vascular Surgery and North American Chapter, International Society for Cardiovascular Surgery. J Vasc Surg. 1991 Mar;13:452-8. PubMed PMID: 1999868.

7. Dillavou ED, Muluk SC, Makaroun MS. Improving aneurysmrelated outcomes: nationwide benefits of endovascular repair. J Vasc Surg. 2006 Mar;43:446-51, discussion 451-2.

8. Prinssen M, Verhoeven ELG, Buth J. A randomized trial comparing conventional and endovascular repair of abdominal aortic aneurysms. ACC Curr J Rev. 2005 Jan;14:59-60.

9. Ohki T, Malas M. Endovascular treatment of abdominal and thoracic aortic aneurysm In: Manual of.Peripheral Vascular Intervention. Lippincott Williams and Wilkins. 2005:206-7.

10. Noel aneurismas de la aorta, Gloviczki P, Cherry KJ, Bower TC, Panneton JM, Mozes GI, et al. Ruptured abdominal aortic aneurysms: The excessive mortality rate of conventional repair. J Vasc Surg. 2001;34:41-6.

11. Criado FJ, Fairman RM, Becker GJ. Talent LPS aneurismas de la aorta abdominal stent graft: Results of a pivotal clinical trial. J Vasc Surg. 2003;37:709-15.

12. Johnston KW1, Rutherford RB, Tilson MD, Shah DM, Hollier L, Stanley JC. Suggested standards for reporting on arterial aneurysms. Subcommittee on Reporting Standards for Arterial Aneurysms, Ad Hoc Committee on Reporting Standards, Society for Vascular Surgery and North American Chapter, International Society for Cardiovascular Surgery. J Vasc Surg. 1991;13:452-8. PubMed PMID: 1999868.

13. Hirsch AT, Haskal ZJ, Hertzer NR, Bakal CW, Creager MA, Halperin JL, et al. ACC/AHA 2005 Practice Guidelines for the management of patients with peripheral arterial disease (lower extremity, renal, mesenteric, and abdominal aortic): a collaborative report from the American Association for Vascular Surgery/Society for Vascular Surgery Society for Cardiovascular Angiography and Interventions, Society for Vascular Medicine and Biology, Society of Interventional Radiology, and the ACC/AHA Task Force on Practice Guidelines (Writing Committee to Develop Guidelines for the Management of Patients With Peripheral Arterial Disease): endorsed by the American Association of Cardiovascular and Pulmonary Rehabilitation; National Heart, Lung, and Blood Institute; Society for Vascular Nursing; TransAtlantic Inter-Society. Consensus; and Vascular Disease Foundation. Circulation. 2006;113:e463-654. Review. PubMed PMID: 16549646.

14. Greenhalgh R. Comparison of endovascular aneurysm repair with open repair in patients with abdominal aortic aneurysm (EVAR trial 1), 30-day operative mortality results: randomised controlled trial. The Lancet. 2004;364:843-8.

15. Diethrich EB. Side branch preservation during endovascular aortic aneurysm repair. J Endovasc Ther. 2001;8:1-2.

16. Greenberg RK, Lu Q, Roselli EE, Svensson LG, Moon MC, Hernandez AV, et al. Contemporary Analysis of Descending Thoracic and Thoracoabdominal Aneurysm Repair: A Comparison of Endovascular and Open Techniques. Circulation. 2008;118:808-17, 19.

17. Greenberg RK, Sternbergh WC, Makaroun M, Ohki T, Chuter $\mathrm{T}$, Bharadwaj P, et al. Intermediate results of a United States multicenter trial of fenestrated endograft repair for juxtarenal abdominal aortic aneurysms. J Vasc Surg. 2009;50:730-70.

18. Gutiérrez J, Zanabili A. Endofugas tipo II tras tratamiento endovascular de los aneurismas de aorta abdominal: incidencia, factores predisponentes, pruebas diagnósticas, indicaciones y alternativas terapéuticas. Angiologia. 2009;61:195-204.

19. Haddad F, Greenberg RK, Walker E, Nally J, OŃeill S, Kolin G, et al. Fenestrated endovascular grafting: The renal side of the story. J Vasc Surg. 2005;41:181-90. 